\title{
Isolated Sacrococcygeal Tuberculosis, A Case Report Of A Rare Atypical Presentation
}

\author{
Daksh Gadi ${ }^{1}$, Saket Nigam ${ }^{2}$, Deepti Gupta ${ }^{3}$,Akanksha Sinha ${ }^{4}$
}

\begin{abstract}
Tuberculosis is a major health problem in India and in view of rise in the MDR tuberculosis atypical presentations are getting commoner these days and are difficult to diagnose. Musculoskeletal tuberculosis is very common among the hematogenous spread cases of pulmonary tuberculosis involving thoracic spine the most. Involvement of sacrum and coccyx is rarest of the possibility and isolated sacrococcygeal tuberculosis thus is very rare. Being a rare entity a high index of clinical suspicion is required to diagnose it. We hereby report a very rare case report of sacrococcygeal tuberculosis.
\end{abstract}

Index Terms-Sacrococcygeal; Spine; Tuberculosis.

\section{INTRODUCTION}

Tuberculosis is a major health problem of developing nations. Pulmonary tuberculosis is the most common site and hematogenous spread has been considered most common type of spread to extrapulmonary sites ${ }^{1}$. Musculoskeletal system being among the $10 \%$ of the extrapulmonary sites of tuberculosis ${ }^{2}$, spine accounts for $50 \%$ among skeletal tuberculosis cases ${ }^{3}$. Although spine involvement is so high still lumbosacral region involvement is just $2-3 \% \%^{3}$. And in the literature most cases of sacral tuberculosis are due to direct spread from lumbar spine $\mathrm{s}^{4}$. To the best of our knowledge very few cases of isolated sacrococcygeal tuberculosis have been described in the literature globally.

\section{CASE REPORT}

A 23year old female presented to us with low back pain and radiation of pain to right gluteal region since 3 months. She complained of slight swelling in right gluteal region. There was no H/O fever, cough, malaise or weight loss. On examination swelling in right gluteal region with mild local rise of temperature in right gluteal region was noted. Hip range of movement was within normal limits. There was no distal neurological deficits and power in bilateral lower limb muscles was $5 / 5$. Perianal and perineal sensations were intact and there was no bladder or bowel involvement.

X-rays of lumbosacral spine showed osseus destruction of sacrococcygeal segment and anterior invert angulation of $\operatorname{coccyx}($ Figure 1,2). A suspicion of osteomyelitis was there

\footnotetext{
1.Daksh Gadi Consultant (Joint replacement) Department of Orthopaedics and Joint Replacement in Regency hospital, Kanpur (dr daksh@yahoo.com)

2.Saket Nigam, Consultant(Interventional Radiologiy) Department of Radiology, Regency Hospital, Kanpur (drsaketnigam@rediff.com) 3.Deepti Gupta, Consultant( Pathology) Department of Pathology, Regency Hospital, Kanpur (guptadeeps@gmail.com)

4.Akanksha Sinha, Consultant (Cornea \& Anterior segment) Sankara eye hospital, kanpur (drakanksha08@gmail.com)
}

so MR scan was done which showed hyperintense signals on T2/STIR s/o marrow edema with minimal adjacent soft tissue suggestive of Infective (osteomyelitis) or tuberculosis (Figure 3,4,5).

On laboratory investigations, $\mathrm{Hb}$ was $10.1 \mathrm{~g} / \mathrm{dl}$, TLC was 11600, Erythrocyte sedimentation rate (ESR) was $90 \mathrm{~mm} / 1$ hour and C-Reactive protein (CRP) was $60 \mathrm{mg} / \mathrm{L}$. Mantoux test was positive with an induration of $15 \times 15 \mathrm{~mm}$. CT guided biopsy was done which revealed fragments of lamellated bony trabeculae with intervening fibrocollagenous tissue showing well formed caseating epithelioid cell granulomas with langhan's giant cells and mononuclear inflammatory infilterate (Figure 6,7). No evidence of any malignancy was observed. $\mathrm{ZN}$ staining revealed no acid fast bacilli. So these finding were consistence of bony destruction due to tuberculosis.

Treatment was started anti-tuberculous four drug regimen for initial intensive phase of three months. Improvement of symptoms i.e. pain in the back and gluteal region was prompt within one month of therapy, Monthly assessment of inflammatory markers (ESR, CRP) was done.

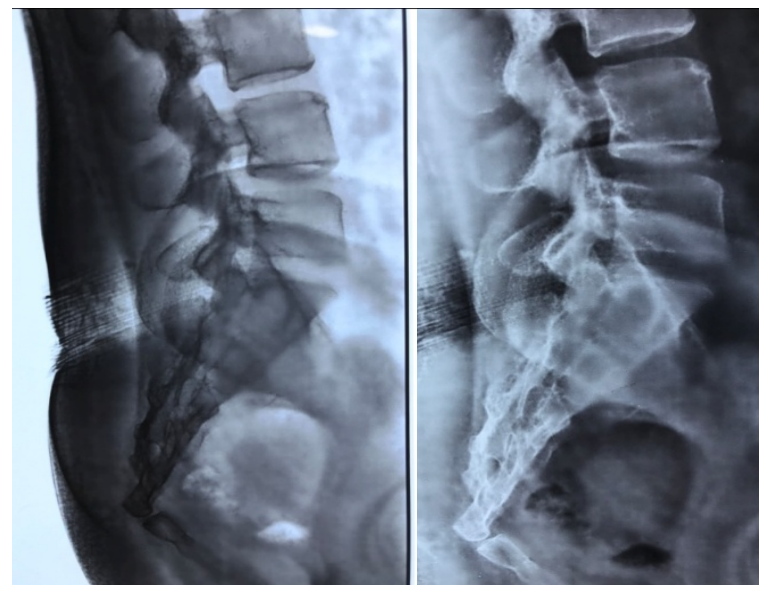

Figure 1 and 2: Xray showing sacrococcygeal osteolysis and anterior inverted coccyx.

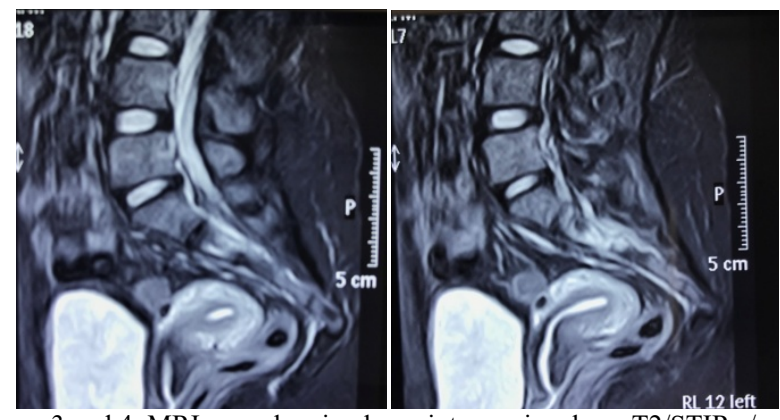

Figure 3 and 4: MRI scan showing hyperintense signals on T2/STIR s/o marrow edema with minimal adjacent soft tissue suggestive of osteomyelitis/tuberculosis 


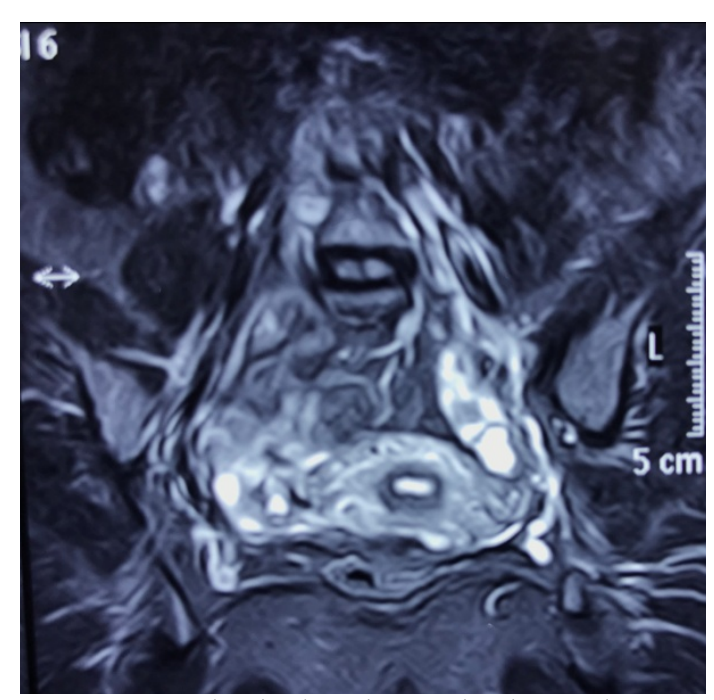

Figure 5: MRI scan showing hyperintense signals on T2/STIR s/o marrow edema with minimal adjacent soft tissue suggestive of osteomyelitis/tuberculosis

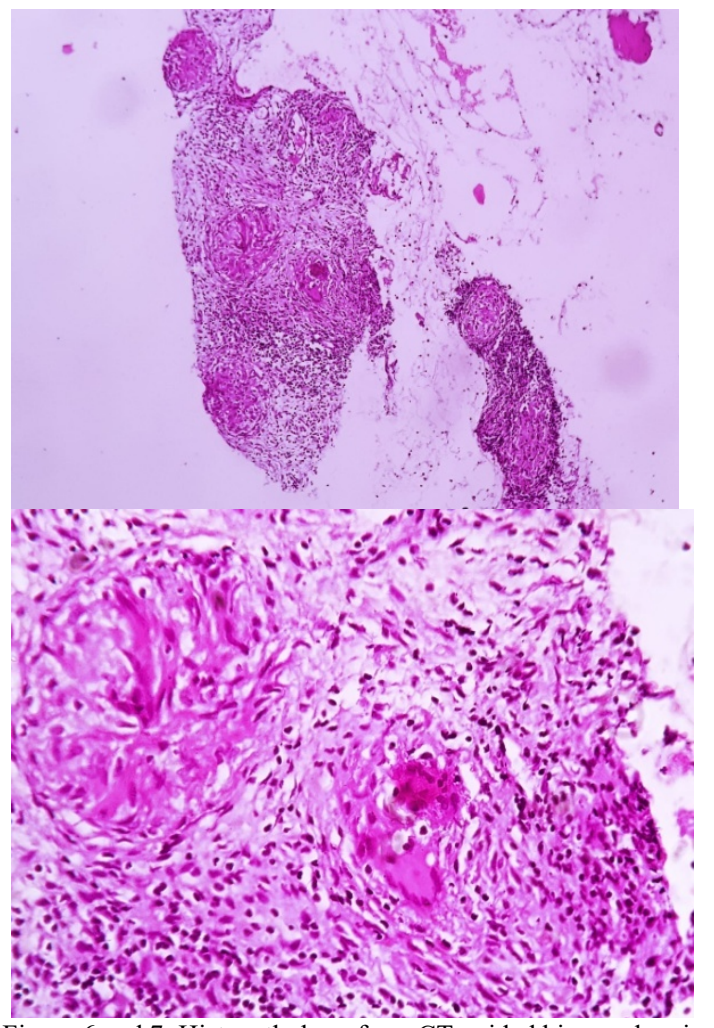

Figure 6 and 7: Histopathology from CT guided biopsy showing caseating epithelioid cell granuloma. detailed history taking is required for diagnosing such cases. Most of the cases are treated with absolute bed rest and analgesics for long time before being diagnosed as tuberculosis. Delay in diagnosis of such cases will lead to progression of the disease and complications like Caudaconus syndrome associated to sacral tuberculosis has also been reported in Indian population ${ }^{7}$.

Such cases may be associated with abscess or anal fistula ${ }^{8}$ so thorough clinical examination and whole spine screening MRI with gluteal and thigh MR cuts may be required. High suspicion of skip lesions should be there in any sacral tuberculosis case. Coccygeal involvement is very rare and to the best of our knowledge no case of coccygeal tuberculosis has been described in literature and in our case edema was noted around coccyx and anterior invert coccyx was seen.

Bone tuberculosis require a treatment of minimum 12 to 18 months for complete healing and a shorter duration may lead to recurrence of infection.

\section{CONCLUSION}

Sacral tuberculosis is a rare entity and has a high chance of delayed diagnosis leading to progression of disease. Neurodeficits are uncommon but may be seen and in any gluteal and low back pain case investigations should include visualisation of sacrococcyx and though rare but a diagnosis of isolated sacrococcygeal tuberculosis should be kept in mind.

\section{ACKNOWLEDGMENT}

I would like to thank Dr. Akanksha Sinha for her valuable guidance in drafting the manuscript, she was involved in collection of data and editing various aspects of the article.

\section{REFERENCES}

[1] Tuli SM. Clinical features. In: Tuli SM, editor. Tuberculosis of skeletal system: bones, joints, spine and bursal sheaths. 2nd ed. New Delhi: Jaypee Brothers; 1997. p.177-82.

[2] Rajasekaran S, Dheenadhyalan J. Tuberculosis of bone and joints. In: Bulstrode C, Buckwalter J, Carr A, et al., editors. Oxford textbook of orthopaedics and trauma. Oxford: Oxford University Press; 2002. p.1532-60.

[3] Cabral MML, Azevedo BCCDA, Montenegro LML et al. Tuberculous spondylitis in teenager. J Bras Pneumol 2005;31 (3) : 261-4.

[4] Campbell WC. An analysis of 51 bone and joint affections treated by heliotherapy, with special reference to tuberculosis. J Bone Joint Surg Am 1917; 2 (15): 1-16.

[5] Ahmadi J, Bajaj A, Destian S, Segall HD, Zee CS. Spinal tuberculosis: atypical observations at MR imaging. Radiology 1993;189:489-93.

First case of sacral tuberculosis was described by Campbell in 1917 and was not an isolated sacral tuberculosis, it was a skip lesion involving both lumbar and sacral spine ${ }^{4}$. Spinal tuberculosis usually has a haematogenous spread through the paravertebral venous Batson plexus from lung or genitourinary tract ${ }^{5}$. A rise in the AIDS and immunodeficiency has lead to an increase in the number of MDR and atypical tuberculosis ${ }^{6}$. Very few isolated sacral tuberculosis has been reported in literature, so being a rare entity a high index of suscpicion and
[6] Radheshyam Sament et al. Asian Spine J 2013;7(4):351-354

[7] VPS Punia, Satish Kumar. JIACM 2008; 9(1): 57-60

[8] Seidel K. Anal fistula cause by sacrococcygeal tuberculosis. Z Tuberk 1950;94:307-9. 